$\xi=$

\title{
MicroRNAs: new biomarker for diagnosis, prognosis, therapy prediction and therapeutic tools for acute lymphoblastic leukemia
}

\author{
Eman A Gobran M. Sc ${ }^{1 *}$, Ghada M Nasr PhD ${ }^{2}$, Mohamed Y Nasr PhD ${ }^{1}$, Mahmoud I Nasr PhD ${ }^{1}$ \\ ${ }^{1}$ Molecular Biology Department, Genetic Engineering and Biotechnology Research Institute (GEBRI), University of Sadat City, Egypt \\ ${ }^{2}$ Molecular Diagnostics Department, Genetic Engineering and Biotechnology Research Institute (GEBRI), University of Sadat City, Egypt \\ *Corresponding author E-mail: emangobran81@gmail.com
}

\begin{abstract}
Back ground: MicroRNA (miRNA) was originally discovered in Caenorhabditis elegans by Victor Ambrose in 1993. It was predicted that miRNA account for 1-6\% of the human genome and regulated at least 33\% of protein-coding genes. Recently, 945 distinct miRNAs molecules have been identified within the human genome. It has been associated with the pathogenesis, progression and prognosis of different diseases, such as leukemia. MiR-181 family is one of those miRNA families, which generally expressed in 70 species and various human cancers.

Objective: Assessment of mir-181a and LDH as promising co-biomarkers in order to provide additional information influence decisions about treatment sequentially to improve health outcomes of ALL in the Egyptian children.

Patients and methods: This study was conducted on 100 children; 50 with ALL (38 males and 12 females) as ALL group. Other 50 healthy age and sex matched children were collected randomly as control group. Serum lactate dehydrogenase (LDH) and miRNA-181a were evaluated for all participants.

Results: The results showed that there was a statistical significant difference between ALL and control groups regarding both molecular and biochemical indications of mir-181a and LDH were about almost five time as the control value, $(\mathrm{P}=0.001)$. The ROC curve analysis revealed that the studied LDH and mir-181a markers were highly sensitive, highly specific and highly accurate test in the differentiation between the two studied ALL and control groups, with cutoff: ALL if mir-181a > 2.071, ALL if LDH > 0.307 respectively. Conclusion: MiRNA-181a and LDH can be used as co-biomarkers for ALL and might be beneficial in early diagnosis.
\end{abstract}

Keywords: ALL; LDH; miRNA-181-a; Real Time-PCR; Leukemia.

\section{Introduction}

Leukemia was classified into myeloid and lymphatic groups and acute leukemia was separated from chronic leukemia based on a rapid development of the disease and a short time between presentation and death. In children, $75 \%$ of cases develop from precursors of the Bcell lineage, with the remainder of cases consisting of malignant T-cell precursors. In spite of these scientific advances, leukemia was a fatal disease, inevitably leading to death (Payandeh et al., 2014). Acute lymphoblastic leukemia (ALL) is the most common cause of cancer in children and adolescents, which affected about 880,000 people and resulted in 110,000 deaths globally in 2015 . The highest rates seen between the ages three and seven years; around $75 \%$ of cases occur before the age of 6 with a secondary rise after the age of 40 (Hunger and Mullighan, 2015). Diagnosis is established by the presence of $20 \%$ or more lymphoblasts in the peripheral blood or bone marrow. Evaluation for morphology, flow-cytometry, Immuno-phenotyping and cytogenetic testing is helpful both for confirming the diagnosis and risk stratification. Great advances in leukemia management during the last 20 years have resulted in high cure rates of more than $80 \%$ of children. Unfortunately, treatment related death for this disease is still 2-4\% (Cooper and Brown, 2015). The hallmark of ALL is genetic alterations and chromosomal abnormalities that involved in the proliferation and differentiation of lymphoid precursor cells. Traditionally, risk stratification has been based on clinical factors such age, white blood cell count and response to chemotherapy; however, the identification of recurrent genetic alterations has helped refine individual prognosis and guide management. Despite advances in management, the backbone of therapy remains multi-agent chemotherapy with vincristine, corticosteroids and an anthracycline with allogeneic stem cell transplantation for eligible candidates. Treatment with arsenic, blood transfusions and radiation led only to short remissions. Elderly patients and/or children are often unable to tolerate such regimens and carry a particularly poor prognosis. For further treatment improvement, it is necessary to customize treatment for each individual patient (Terwilliger and Abdul-Hay, 2017).

Lactic dehydrogenase (LDH) is a pyridine-linked enzyme found in almost all animals' tissues, functions essentially in the metabolism of glucose, catalyzing the reduction of free pyruvate to lactate during the last step of glycolyis, as well as the conversion of lactate to pyruvate during gluconeogenesis. Malignant cells have a distinctive type of metabolism in which the glycolytic sequence and the tricarboxylic acid cycle are poorly integrated; hence, the cells tend to consume from five to ten times as much glucose as do normal tissues, converting most of it into lactate. Whether the increased serum levels of LDH frequently found in cancer patients reflect greater production 
and release of the enzyme by malignant cells or alterations in the metabolic interplay among organs in which LDH exists in high concentrations, or perhaps a combination of the two (i.e. greater production and release of the enzyme and alterations in the metabolic interplay among organs) (Liu et al., 2016).

In 1961, Jacob and Monod reported that some tiny RNA molecules can bind to messenger RNAs (mRNAs) through base-pairing and therefore inhibit mRNA expression. No one could imagine that in the following decades these small non-coding RNAs would open a new chapter in our understanding of how the expression of genes is being regulated (Lee and Young, 2013). In 1993, when 21 base pair long RNAs were originally discovered in Caenorhabditis elegans, and then these microRNAs (miRNAs), latterly found in most eukaryotes including humans (Younis et al., 2014). It is predicted that miRNA account for 1-6\% of the human genome and regulate at least 33\% of protein-coding genes. To date, 945 distinct miRNAs molecules have been identified within the human genome. Although little is currently known about the biological functions and specific targets of miRNA molecules thus far, it is evident that miRNA plays a critical role in the regulation of gene expression that controlling diverse cellular and metabolic pathways. Thus, it has been associated with the pathogenesis, progression and prognosis of different diseases, such as leukemia. Scientists have gained more in-depth knowledge of the basic mechanism of action of miRNAs, but the main challenge still remaining to identify the direct targets of these important "microplayers", in order to understand how they work in many biological environments in both healthy and diseased tissues. Many technologies have been improved in the past few years, some with more potential than others, but all with their own pros and cons (Trzybulska et al., 2018).

Like protein coding genes, miRNA sequences can be grouped into families and the relationship between their structures and functions can be learnt from multiple sequence alignments in miRNA families (Griffiths-Jones et al., 2013). A miRNA family usually has several members which are different in 1-2 nucleotides only. MiR-181 family is one of those miRNA families, which generally express in 70 species and various human cancers. This family includes 4 members (miR-181a, miR-181b, miR-181c and miR-181d) and they are highly conserved in the seed-region sequence and RNA secondary structure. Among them, miR-181a and miR-181b (miR-181a/b) which locate on the same loci of chr 1q31.3 and chr 9q3.33 are the most studied (Kozomara and Griffiths-Jones, 2011).

Ciafre et al., 2005 firstly reported that expression of $\mathrm{miR}-181 \mathrm{a} / \mathrm{b}$ was significantly downregulated in primary glioblastomas and human glioblastoma cell lines compared to normal brain tissue, by using microarray and northern blot analysis. Thereafter, miR-181a/b was discovered abnormally expressed in various malignant cells including both solid tumors and hematological malignancies. As in glioblastoma, a significant down-regulation of miR-181a level was also observed in squamous lung cell carcinoma (SQCC), oral squamous cell carcinoma (OSCC) and non-small-cell lung cancer (NSCLC) (Gao et al., 2010 ; Gae et al., 2011). However, miR-181a was significantly over-expressed in MCF-7 breast cancer cells and hepatocellular carcinoma (HCC) cells. Other studies also reported that miR-181a had different expression levels in hematological malignancies. It is upregulated in acute myeloid leukemia (AML), especially in M1 and M2 subtypes, and myelodysplastic syndromes (MDS), but down-regulated in multiple myeloma (MM) and chronic lymphocyte leukemia (CLL). miR-181a/b might co-express and play critical roles together as a prognosis factors in human cancers (Kaur et al., 2011).

Detailed analysis of human blood cells revealed a high expression of miR-181a in hematopoietic stem cells (HSCs); whereas expression in mature hematopoietic cells was considerably lower (Landgraf et al., 2014). Two studies investigated the role of miR-181a during Tcell development and activation markers Cluster of Differentiation 5 (CD5) and CD69 as direct targets of miR-181a and described an inhibiting role of miR-181a in T-cell activation (Li et al., 2014 ; Neilson et al., 2014). However, the linkage between miRNAs especially mir-181 family and susceptibility to Acute Lymphoblastic Leukemia has not been well-established. In the present study, we aimed to assess mir-181a as a promising genetic biomarker in order to provide additional information influence decisions about treatment sequentially to improve health outcomes of ALL in the Egyptian children.

\section{Patients and methods}

\subsection{Study population}

This case-control study was conducted in Molecular Biology Department and Molecular Diagnostics Department, Genetic Engineering and Biotechnology Research Institute, Sadat City University, Egypt; in collaboration with Medical Biochemistry and Molecular Biology Department, Faculty of Medicine, Ain Shams University, Egypt. It included $100 \quad$ children subdivided into two groups-group 1: a total of 50 newly diagnosed ALL patients (38 males and 12 females, with mean of age $5.63 \pm$ 2.34 years); group 2: a total of 50 normal healthy age and sex matched children ( 25 males and 25 females, with mean of age $6.22 \pm 2.20$ years) were collected randomly from the period of February to December 2018.

Full history taking, thorough clinical examination and routine investigations, bone marrow examination, cytochemistry and immunophenotyping were performed for patients to confirm diagnosis and classification. A written informed consent was signed by the parents of each child. The study was approved by the Research Ethics Committee of Sadat City University and Ain Shams University Hospitals, Egypt.

\subsection{Sample collection and processing}

Venous blood $(6 \mathrm{~mL})$ was withdrawn from each subject under complete aseptic conditions and was left to clot for 20 min. Serum was separated by centrifugation at $4000 \mathrm{~g}$ for $10 \mathrm{~min}$. All samples were kept at $-80^{\circ} \mathrm{C}$ until use.

\subsection{Quantitative determination of serum LDH}

The quantitative determination of serum LDH was done by spectrophotometer Cobas C111 using the commercially available Optimized test according to German Society of Clinical (DGKC) supplied by (RANDOX, 55 Diamond, Crumlin, Country Antrim, BT294QY, United Kingdom); according to the manufacturer's instructions www.randox.com.

\subsection{Relative expression of miRNA 181-a using quantitative real time polymerase chain reaction}

Total RNA including miRNA was extracted from sera using MagNA Pure Compact Nucleic Acid Isolation Kit I (Cat. no. 03730964001) supplied by (Roche, Germany), according to the magnetic particle with glass fiber affinity protocol (Cook et al., 2004). Then, the total 
RNA was reverse transcribed using EPIK ${ }^{\mathrm{TM}}$ miRNA Select protocol. Expression of miRNA 181 -a and its housekeeping gene $\beta$ actin (used as endogenous control) was measured by quantitative real time polymerase chain reaction (qPCR) performed by (Real-time 7500 Fast PCR System; applied bio systems) using SensiSMART SYBR Master Mix according to the manufacturer's protocol. Relative expression levels of the miRNA-181a were measured using $2^{-\Delta \Delta C t}$ method (Livak and Schmittgen, 2001) with $\beta$ actin being used as the internal control to normalize the data. Finally, perform qPCR amplification curve (Fig. 1).

The miRNA 181-a Forward Probe Primers Sequence were:

5' AGATCTAGCCCAATATCGGCCATGTT 3'

Reverse Primers were:

5' CTCGAGAGAAAGTCCTGGTGTGTCCA 3'

The $\beta$-actin Forward Probe Primers Sequence were:

5'CAGAGCCTCGCCTTTGCC 3'

Reverse Primers were:

5'GTCGCCCACATAGGAATC $3^{\prime}$

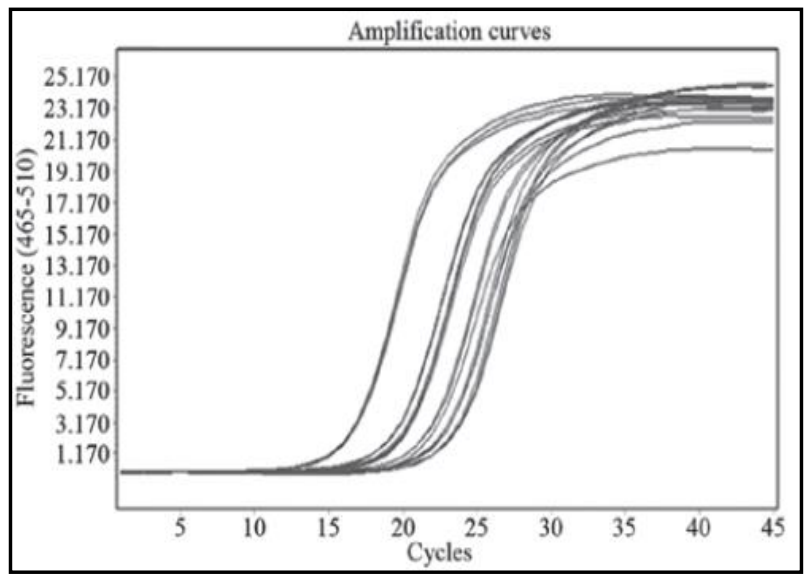

Fig. 1: Amplification Plot (Rn vs. Cycle) of miRNA 181-a.

\subsection{Statistical analysis}

All analyses were done using the Statistical Package for the Social Sciences (SPSS software version 20, Chicago, IL, USA) on a personal computer. Data are expressed as mean \pm SD or percentage $(\%)$. Test of normality, Kolmogorov-Smirnov test, was used to measure the distribution of data. Accordingly data are not normally distributed, so comparison between variables in the two groups was performed using Mann Whitney test. Receiver operating curve (ROC) test was used discriminate between diseased (ALL) and control groups and to calculate the diagnostic indices (sensitivity, specificity, positive and negative predictive values and accuracy) of both micro-RNA and LDH.

\section{Results}

\subsection{Demographic and hematological laboratory parameters of study subjects}

The demographic features showed that the mean age of ALL patients was $5.63 \pm 2.34$ years and the majority of the patients were males (38 patients, 76\%) (Table 1). The hematological parameters showed that there was a statistical significant difference between ALL and control groups regarding the hematological founding of the complete blood picture (CBC), erythrocyte sedimentation rate (ESR) and Creactive protein $(\mathrm{P}=0.001)$; mean cell hemoglobin $(\mathrm{MCH})$ and mean cell hemoglobin concentration $(\mathrm{MCHC})$ were exceptions; $(\mathrm{P}=$ $0.970,0.224$ respectively) (Table 2 ).

Table 1: Demographic Features in the Two Studied Groups

\begin{tabular}{lll}
\hline & Control $(\mathrm{n}=50)$ & ALL $(\mathrm{n}=50)$ \\
\hline Age (yrs.) & $6.22 \pm 2.20$ & $5.63 \pm 2.34$ \\
Gender & & \\
Female & $25(50.0 \%)$ & $12(24.0 \%)$ \\
Male & $25(50.0 \%)$ & $38(76.0 \%)$ \\
\hline
\end{tabular}

Table 2: Complete Blood Picture in the Two Studied Groups

\begin{tabular}{|c|c|c|c|}
\hline & Control $(n=50)$ & $\operatorname{ALL}(n=50)$ & $\mathrm{P}$ value \\
\hline RBCs & $4.74 \pm 1.17$ & $2.91 \pm 0.78$ & $0.001 *$ \\
\hline $\mathrm{Hb}$ & $11.85 \pm 0.54$ & $7.45 \pm 2.17$ & $0.001 *$ \\
\hline Het & $36.00 \pm 1.53$ & $22.95 \pm 5.64$ & $0.001 *$ \\
\hline $\mathrm{MCV}$ & $78.55 \pm 1.82$ & $79.66 \pm 6.31$ & $0.048^{*}$ \\
\hline $\mathrm{MCH}$ & $25.90 \pm 0.65$ & $25.68 \pm 3.45$ & 0.970 \\
\hline $\mathrm{MCHC}$ & $32.99 \pm 1.02$ & $32.29 \pm 4.48$ & 0.224 \\
\hline Platelets & $341.60 \pm 48.60$ & $132.92 \pm 107.60$ & $0.001 *$ \\
\hline WBCs & $9.47 \pm 1.55$ & $28.02 \pm 20.53$ & $0.001 *$ \\
\hline ESR $1^{\text {st }} \mathrm{hr}$. & $4.18 \pm 1.70$ & $62.18 \pm 18.06$ & $0.001 *$ \\
\hline $\mathrm{ESR} 2^{\text {nd }} \mathrm{hr}$. & $10.42 \pm 4.00$ & $93.96 \pm 17.89$ & $0.001 *$ \\
\hline CRP & $3.28 \pm 1.44$ & $62.56 \pm 54.86$ & $0.001 *$ \\
\hline
\end{tabular}

Data are expressed as mean \pm SD $\mathrm{P}>0.05=$ not significant $* \mathrm{P} \leq 0.05=$ significant. 


\subsection{Serum miRNA 181-a RQ values and LDH levels in ALL patients}

Results showed that serum miRNA 181-a RQ values and LDH levels were significantly higher in ALL group compared with control group $(\mathrm{P}<0.001$, Table 3$)$. In addition, regression analysis of receiver operating curve (ROC) revealed that the studied LDL and mir-181a markers were highly sensitive, highly specific and highly accurate test in the differentiation between the two studied ALL and control groups with cutoff: ALL if mir-181a > 2.071; ALL if LDH > 0.307 (Table 4 ; Fig. 2, 3).

Table 3: Molecular and Biochemical Indications in the Two Studied Groups

\begin{tabular}{llll}
\hline & Control $(\mathrm{n}=50)$ & ALL $(\mathrm{n}=50)$ & P value \\
\hline LDH & $281.72 \pm 16.67$ & $903.54 \pm 356.89$ & $0.001^{*}$ \\
MiRNA 181-a & $0.48 \pm 0.44$ & $3.87 \pm 1.40$ & $0.001^{*}$ \\
\hline
\end{tabular}

Table 4: Diagnostic Indices of miRNA 181-a RQ and LDH Levels in ALL Group

\begin{tabular}{lll} 
& Table 4: Diagnostic Indices of miRNA 181-a RQ and LDH Levels in ALL Group & \multicolumn{1}{c}{ LDH } \\
\hline & MiRNA 181-a & $>0.307$ \\
\hline Cutoff & $>2.071$ & 0.964 \\
Area under ROC & 0.995 & $90.0 \%$ \\
Sensitivity & $94.0 \%$ & $98.0 \%$ \\
Specificity & $96.0 \%$ & $97.8 \%$ \\
Positive predictive value (PPV) & $95.9 \% \%$ & $90.7 \%$ \\
Negative predictive value (NPV) & $94.1 \%$ & $94.0 \%$ \\
Accuracy & $96.0 \%$ & \\
\hline
\end{tabular}

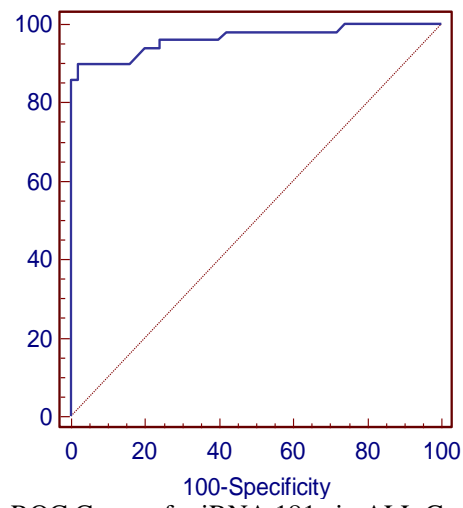

Fig. 2: ROC Curve of miRNA 181a in ALL Group.

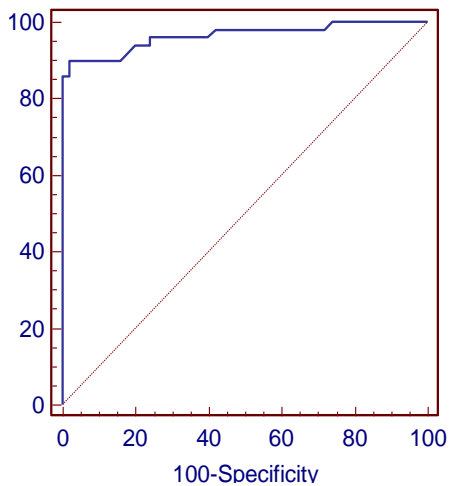

Fig. 3: ROC Curve of LDH in ALL Group.

\section{Discussion}

The magnitude of the cancer problem remained unknown until recently when Egypt National Cancer Registry Program published its results in 2014 (Ibrahim and Nazmi, 2016). Acute lymphoblastic leukaemia, a malignant disorder of lymphoid progenitor cells, affects both children and adults, with peak prevalence between the ages of 2 and 5 years. Steady progress in development of effective treatments has led to a cure rate of more than $80 \%$ in children. Advances in our understanding of the pathobiology of ALL; fuelled by emerging molecular technologies, suggest that the management specifically targeting the genetic defects of leukaemic cells could improve the treatment of this disease.

It is important to study miRNA which is small (17-25 nucleotides) single-stranded non-coding RNAs that function predominantly as sequence-targeted modifiers of gene expression through translational repression. The miRNA plays an essential role in the control of normal hematopoiesis and their disruption may contribute to leukemogenesis. Few studies have been conducted until now concerning the role of miRNA in childhood ALL. These studies have suggested that miRNA expression analysis can help to understand the development of several phenotypes and biological functions of these miRNAs in childhood ALL (Vasilatou et al., 2010 ; Wang et al., 2015).

Lactate dehydrogenase is an enzyme, consisting of five different isoenzymes which catalyze the interconversion of L-lactate and pyruvate. LDH is present in the cytoplasm of all human tissues with higher concentrations in heart, liver and skeletal muscle, and lower values in erythrocytes, pancreas, stomach and kidney. Increased LDH activities are found in a variety of pathological conditions such as myocardial infarction, diseases of liver, blood, muscle or cancer. The current work aimed to evaluate miRNA-181a and LDH as a promising co-biomarker in order to provide additional information influence decisions about treatment sequentially to improve health outcomes of ALL in the Egyptian children. 
In this study, males constituted $76 \%$ of ALL patients. Accordingly, higher incidence of ALL was observed in males as reported by other studies (Rana et al., 2015; Lee and Cho, 2017). There was a statistical significant difference between ALL and control groups regarding hematological founding of the complete blood picture $(\mathrm{CBC})(\mathrm{P}=0.001)$; although $\mathrm{MCH}$ and $\mathrm{MCHC}$ were exception; $\mathrm{P}=0.970,0.224$ respectively. This may be explained as levels for $\mathrm{MCH}$ and $\mathrm{MCHC}$ are tracked for ALL as MCH levels are the average amount of hemoglobin that is in each red blood cell; MCHC levels are the average weight of that hemoglobin based on the volume of red blood cells. Both are a reflection of the health of the hemoglobin in the blood. These results were in accordance with these of El-Shafey et al., 2017 who compared the hematological founding in Egyptian children with Acute Lymphoid and Myeloid leukemia and found that there were a significant difference between the blood indices of CBC in the studied ALL children during and after chemotherapy comparing with controls $(\mathrm{P}<0.001)$ except for MCH and MCHC, $(\mathrm{P}=0.416)$. In contrast, Jaime-Pérez et al., 2019 who studied the CBC and clinical findings at diagnosis of childhood ALL and found that all blood cells and the blood indices showed a significant difference between the ALL group and the controls group $(\mathrm{P}<0.001)$.

$\mathrm{C}$-reactive protein is a marker of acute phase inflammatory response. It is produced mainly by hepatocytes, and its production is regulated by interleukin 6 (IL-6). Both genetic and environmental factors influence an individual's basal CRP concentration. Increased CRP concentrations have been reported in many diseases, including cardiovascular diseases, type 2 diabetes, arthritis and many types of cancers. ESR; another inflammatory marker showed an increase with cancer progression indicating the presence and influence of chronic inflammation in the pathogenesis of this disease (Rasic et al., 2016). There was a statistical significant difference between ALL and control groups regarding other hematological parameters including ESR and serum CRP, $(P=0.001)$. These results can be interpreted as moderately elevated ESR occurs with inflammation and also with anemia; where a very high ESR usually has an obvious cause, such as a severe infection. Also, CRP has been traditionally utilized as a marker of infection and inflammation. These results were in agreement with that of Starke et al., 2010 who studied both ESR and serum CRP levels in the management of infection in ALL and found a significant difference between patients and controls $(\mathrm{P} \leq 0.05)$. On the other hand Ishii et al., 2010 who studied the fever in children with acute leukemia and found that there was no significant difference between CRP or ESR in ALL patients compared with control during fever indicating that CRP and ESR didn't have a rule in the duration of ALL disease.

In the current study there was a statistical significant difference between ALL and control groups regarding both molecular and biochemical indications of mir-181a RQ and LDH were about almost five time the control value, $(\mathrm{P}=0.001)$. Increased serum levels of LDH in cancer patients reflect greater production and release of the enzyme by malignant cells or alterations in the metabolic interplay among organs in which LDH exists in high concentrations. However, miRNA-181a RQ was significantly up-regulated in the ALL group as it may be linked to high levels of minimal residual disease and poor prognosis. These results were in agreement with that of Hafiz and Mannan, 2007 who studied serum LDH level in childhood ALL and found that it was about almost six times as the control value (P < 0.001). However, Youssef et al., 2018 who studied the assessment of the metabolic mediators such as LDH in malignant cells and found that only significant high level found in the advanced stages (IV stage) of the disease and non significant different with early stages (I, II and III) as well as control subjects. Also, Wang et al., 2015 who studied the expression and function of mir-181a in children with ALL and found that the ALL group were higher by six times than the control group $(\mathrm{P}=0.001)$. However, when Youssef et al., 2018 linked mir-181a with metabolic mediators such as LDH; he found that miR-181a reprograms the metabolism of malignant cells by increasing glucose uptake and lactate secretion required for increased cancer cell development and proliferation. Also, he reported that in accordance with a pro-cancerous role for miR-181a; a progressive stage and grade-associated highly significant elevated levels of miR-181a in cancers' patients compared to control subjects before therapy. Whereas they couldn't link miRNA- 181a to the prognosis in ALL; the marked decrease in their expression after therapy could reflect the impact on disease outcome.

The present study showed that the studied LDH and mir-181a markers were highly sensitive, highly specific and highly accurate test in the differentiation between the two studied ALL and control groups, with cutoff: ALL if mir-181a RQ > 2.071; ALL if LDH > 0.307. Diagnostic accuracy comparative analysis revealed that serum LDH had the highest sensitivity and specificity ( $90 \%$ and $98 \%)$ respectively, followed by plasma miR-181a (94\% and 96\%). Also, Youssef et al., 2018 reported that serum LDH had the highest sensitivity and specificity (96\% and 95\%) respectively, followed by plasma miR-181a (95\% and 92\%), both markers had the best association with elevated stages and grades compared to all other markers.

\section{Conclusion}

In conclusion, this study suggests that circulating miRNA-181a level could be used as a molecular marker for ALL and it might be advantageous in early diagnosis. Serum LDH level could be used as a biochemical marker for these patients and it could be helpful in early diagnosis. Thus, miRNA-181a and LDH could be used as co-biomarkers for early diagnosis, prognosis and follow-up of ALL. Finally, it is promising that the ability of using miRNA-181a beside LDH as a potential therapeutic targeting for some cancers.

\section{Recommendations}

It is worth mentioning that our data might concur and disagree with some reports. So, we believe that these discrepancies could be attributed to the patient's individual biological differences, pathological staging and grading variation and the sample size effect or all combined together. Therefore, further large scale studies including more number of patients with different stages of disease and more restricted clinicopathological classification are needed to be performed.

\section{References}

[1] Hunger SP, Mullighan CG. Acute Lymphoblastic Leukemia in Children. New England Journal of Medicine. 2015 ; 373 (16): 1541-1552. https://doi.org/10.1056/NEJMra1400972.

[2] Terwilliger T, Abdul-Hay M. Acute lymphoblastic leukemia: a comprehensive review and 2017 update. Blood cancer journal. $2017 ; 7(6)$ : e577. https://doi.org/10.1038/bcj.2017.53.

[3] Payandeh M, Sadeghi E, Khodarahmi R, Sadeghi M. Appearance and disappearance of chronic myeloid leukemia (CML) in patient with chronic lymphocytic leukemia (CLL). International journal of hematology-oncology and stem cell research. 2014; 8(4): 49.

[4] Liu R, Cao J, Gao X, Zhang J, Wang L, Wang B, Wang Z. Overall survival of cancer patients with serum lactate dehydrogenase greater than 1000 IU/L. Tumor Biology. 2016; 37(10): 14083-14088. https://doi.org/10.1007/s13277-016-5228-2.

[5] Trzybulska D, Vergadi E, Tsatsanis C. miRNA and other non-coding RNAs as promising diagnostic markers. EJIFCC. 2018; 29 (3): 221. 
[6] Lee TI, Young RA. Transcriptional regulation and its misregulation in disease. Cell. 2013; 152(6), 1237-1251. https://doi.org/10.1016/j.cell.2013.02.014.

[7] Younis A, Siddique MI, Kim CK, Lim KB. RNA interference (RNAi) induced gene silencing: a promising approach of hi-tech plant breeding. International journal of biological sciences. 2014; 10(10): 1150. https://doi.org/10.7150/ijbs.10452.

[8] Griffiths-Jones S, Bateman A, Marshall M, Khanna A, Eddy SR. Rfam: an RNA family database. Nucleic Acids Res. 2013; 31: 439-441. https://doi.org/10.1093/nar/gkg006.

[9] Kozomara A, Griffiths-Jones S. miRBase: integrating microRNA annotation and deep-sequencing data. Nucleic Acids Res. 2011; 39: D152-157. https://doi.org/10.1093/nar/gkq1027.

[10] Ciafre SA, Galardi S, Mangiola A, Ferracin M, Liu CG. Extensive modulation of a set of microRNAs in primary glioblastoma. Biochem Biophys Res Commun. 2005; 334: 1351-1358. https://doi.org/10.1016/j.bbrc.2005.07.030.

[11] Gao W, Shen H, Liu L, Xu J, Shu Y. MiR-21 overexpression in human primary squamous cell lung carcinoma is associated with poor patient prognosis. J Cancer Res Clin Oncol. 2011; 137: 557-566. https://doi.org/10.1007/s00432-010-0918-4.

[12] Gao W, Yu Y, Cao H, Shen H, Li X. Deregulated expression of miR-21, miR-143 and miR-181a in non-small cell lung cancer is related to clinicopathologic characteristics or patient prognosis. Biomed Pharmacother. 2010; 64: 399-408. https://doi.org/10.1016/j.biopha.2010.01.018.

[13] Kaur P, Bartels CL, Bentley HA, Tsongalis GJ. Select microRNA profiles of patients with chronic lymphocytic Leukemia. Laboratory Investigation. 2011; 91: 450A.

[14] Landgraf P, Rusu M, Sheridan R, Sewer A, Iovino N, Aravin A. A mammalian microRNA expression atlas based on small RNA library sequencing. Cell. 2014; 129(7): 1401-1414. https://doi.org/10.1016/j.cell.2007.04.040.

[15] Li Q, Liu L, Li W. Identifcation of circulating microRNAs as biomarkers in diagnosis of hematologic cancers: a meta-analysis. Tumour Biol. 2014; 35(10): 10467-10478. https://doi.org/10.1007/s13277-014-2364-4.

[16] Neilson JR, Zheng GX, Burge CB, Sharp PA. Dynamic regulation of miRNA expression in ordered stages of cellular development. Genes Dev. 2014; 21(5): 578-589. https://doi.org/10.1101/gad.1522907.

[17] Cook L, Ng KW, Bagabag A, Corey L, Jerome KR. Use of the MagNA pure LC automated nucleic acid extraction system followed by real-time reverse transcription-PCR for ultrasensitive quantitation of hepatitis C virus RNA. Journal of clinical microbiology. 2004; 42(9):4130-4136. https://doi.org/10.1128/JCM.42.9.4130-4136.2004.

[18] Livak KJ, Schmittgen TD. Analysis of relative gene expression data using real-time quantitative PCR and the 2(-Delta Delta C(T)) Method. Methods. 2001; 25(4): 402-408. https://doi.org/10.1006/meth.2001.1262.

[19] Vasilatou D, Papageorgiou S, Pappa V, Papageorgiou E, Dervenoulas J. The role of microRNAs in normal and malignant hematopoiesis. Eur J Haematol, 2010; 84:1-16. https://doi.org/10.1111/j.1600-0609.2009.01348.x.

[20] Ibrahim AS, Nazmi N. Cancer: The Growing Monster in Egypt. J Cancer Prev Curr Res. 2016; 6(4): 00217. https://doi.org/10.15406/jepcr.2016.06.00217.

[21] Lee JW, Cho B. Prognostic factors and treatment of pediatric acute lymphoblastic leukemia. Korean journal of pediatrics. 2017; 60(5): 129. https://doi.org/10.3345/kjp.2017.60.5.129.

[22] Rana ZA, Rabbani MW, Sheikh MA, Khan AA. Outcome of childhood acute lymphoblastic leukaemia after induction therapy-3 years' experience at a single paediatric oncology centre. Journal of Ayub Medical College Abbottabad. 2015; 21(4), 150-153.

[23] El-Shafey AS, Amer SM, Allam NG, El-Alfy MS. Hematological studies in Egyptian children with Acute Lymphoid and Myeloid leukemia. Int. J. Adv. Biomed. 2017; 2(1): 19-29.

[24] Jaime-Pérez, JC, García-Arellano G, Herrera-Garza JL., Marfil-Rivera LJ, Gómez-Almaguer, D. Revisiting the complete blood count and clinical findings at diagnosis of childhood acute lymphoblastic leukemia: 10-year experience at a single center. Hematology, transfusion and cell therapy. 2019; 41(1): 57-61.

[25] Rasic I, Radovic S, Aksamija G. Relationship between chronic inflammation and the stage and histopathological size of colorectal carcinoma. Medical Archives. 2016; 70(2): 104. https://doi.org/10.1016/j.htct.2018.05.010.

[26] Starke ID, De Beer FC, Donnelly JP, Catovsky D, Goldman JM, Galton DA, Pepys MB. Serum C-reactive protein levels in the management of infection in acute leukaemia. European Journal of Cancer and Clinical Oncology. 2010; 20(3): 319-325. https://doi.org/10.1016/02775379(84)90076-2.

[27] Ishii E, Ueda K, Akazawa K, Nose Y. Fever in Children with Acute Leukemia: Cause and Role of Febrile Episode at Initial Diagn osis. Pediatric hematology and oncology. 2010; 7(1), 109-112. https://doi.org/10.3109/08880019009034324.

[28] Hafiz G, Mannan MA. Serum lactate dehydrogenase level in childhood acute lymphoblastic leukemia. Bangladesh Med Res Counc Bull. 2007; 33 : 88-91. https://doi.org/10.3329/bmrcb.v33i3.1139.

[29] Youssef AI, Ahmed MA, Samir M, Mansour IM, El Naggar MA, El Sewedy TS. Assessment of the Metabolic Mediators: Fatty Acid Synthase, Lactate Dehydrogenase and miR-181a as Potential Diagnostic Markers in Colorectal Cancer Patients. Bull. Egypt. Soc. Physiol. Sci. 2018; 38(1):112. https://doi.org/10.21608/besps.2018.8098.

[30] Wang L, Liu X, Peng H, Xu L. The expression and functional study of miR-181a in pediatric acute lymphoblastic leukemia. Zhonghua xueyexue zazhi. 2015; 36(1), 53-57. 